\title{
EXPLORACIÓN DEL SEGMENTO ANTERIOR MEDIANTE TOMOGRAFÍA ÓPTICA DE COHERENCIA 3
}

\section{EXPLORATION OF THE ANTERIOR SEGMENT BY OPTICAL COHERENCE TOMOGRAPHY-3}

\author{
LARA-MEDINA FJ ${ }^{1}$, ISPA-CALLÉN MC ${ }^{1}$, NÚÑEZ A ${ }^{1}$, LÓPEZ-ROMERO $\mathrm{S}^{1}$, \\ LÓPEZ-MONDÉJAR E ${ }^{1}$, ZARCO JM ${ }^{1}$, GONZÁLEZ DEL VALLE F ${ }^{1}$
}

\begin{abstract}
RESUMEN
Objetivo: Dar a conocer la utilidad de la Tomografía de Coherencia Óptica (OCT) para el estudio de patología del segmento anterior así como presentar sus posibles ventajas frente a la biomicroscopía ultrasónica (BMU).

Material y métodos: Presentamos 5 pacientes con diversas patologías del segmento anterior estudiados mediante OCT: 2 pacientes con ataque agudo de glaucoma de ángulo estrecho, 1 paciente con rubeosis iridis, 1 paciente con un traumatismo ocular penetrante y un paciente con un quiste estromal primario del iris.

Resultados: En los pacientes con ataque agudo de glaucoma, el OCT ha mostrado ser una herramienta tan útil como la BMU para la detección del cierre angular. Además, la tomografía óptica fue más cómoda y rápida en la obtención de las imágenes que la BMU, así la el tiempo medio de exploración con OCT suele ser inferior a 5 minutos, a diferencia de la BMU cuyo tiempo suele ser superior a 10 minutos. El OCT también ha demostrado ser una prueba útil para el estudio de otras patologías del iris, permitiendo valorar la permeabilidad de las iridotomías, mostrar neovascularización iridiana e incluso estudiar masas iridianas como el quiste
\end{abstract}

\begin{abstract}
Purpose: To show the utility of optical coherence tomography (OCT) for studying the anterior segment and to explain its potential advantages as compared to ultrasonic biomicroscopy (UBM).

Materials and methods: We have described the findings in 5 patients with different pathologies of the anterior segment, all of whom were studied with UBM and OCT- 3 adjusted for the anterior segment. There were 2 cases of an acute attack of closed angle glaucoma, 1 case of rubeosis iridis, 1 case of penetrating ocular trauma and 1 case of a primary stromal iris cyst. Results: OCT was found to be as useful as UBM in detecting angle closure in patients affected by an acute attack of glaucoma. In addition, OCT was more comfortable for the patient and faster than UBM in obtaining images, with the exploration mean time using OCT being less than five minutes while that with UBM was over ten minutes. OCT has also been demonstrated to be a safe and valuable non-contact examination in other iris pathologies such as rubeosis iridis, for checking the permeability of iridotomies and even for studying iris masses such as primary stromal iris cysts. In these entities OCT may reach a higher resolution than ultrasonic biomicroscopy.
\end{abstract}

Recibido: 16/3/06. Aceptado: 16/11/06.

Complejo Hospitalario La Mancha-Centro, Servicio de Oftalmología, Alcázar de San Juan. Ciudad Real. España.

1 Licenciado en Medicina.

Comunicación presentada en la Reunión Anual de la Sociedad Oftalmológica de Madrid (2005).

Los autores manifiestan que no tienen interés comercial ni han recibido apoyo económico para la realización del presente trabajo.

Correspondencia:

Francisco Javier Lara Medina

Complejo Hospitalario La Mancha-Centro

13600 Alcázar de San Juan (Ciudad Real)

España

E-mail: flarmed@hotmail.com 
estromal de iris. En estos casos, el OCT permite la obtención de imágenes con una mayor resolución espacial que la BMU.

Conclusiones: El OCT 3 mediante el ajuste del enfoque puede servir para obtener imágenes del ángulo camerular así como de otras estructuras del segmento anterior ayudando así a establecer determinados diagnósticos de forma no invasiva, cómoda y segura para el paciente.

Palabras clave: Glaucoma agudo, cierre angular, biomicroscopía ultrasónica (BMU), tomografía de coherencia óptica (OCT), iridotomía, quiste estromal primario de iris.
Conclusion: OCT-3, with an adjustable focus, can obtain images from the scleral angle, as well as from other ocular structures like the iris, thus assisting in the diagnosis of numerous pathologies (Arch Soc Esp Oftalmol 2006; 81: 647-652).

Key words: Acute glaucoma, closure angle, ultrasonic biomicroscopy (UBM), optical coherence tomography (OCT), iridotomy, primary iris stromal cyst.

mediante el ajuste del enfoque de la imagen sobre las estructuras del polo anterior.

\section{SUJETOS, MATERIAL Y MÉTODOS} considera la prueba de elección en el estudio de la anatomía y morfología del ángulo esclero-corneal.

En los últimos años, la aparición de la biomicroscopía ultrasónica (BMU) ha supuesto una mejora en la visualización de la cámara anterior, permitiendo un alto nivel de resolución espacial y una capacidad objetiva de medición de las distintas estructuras angulares (1).

La BMU es una técnica de exploración de contacto, con un sistema de inmersión, que requiere una posición supina de los pacientes. La técnica puede ser poco confortable para algunos pacientes y en ocasiones poco tolerada. Además, la posición de la sonda ecográfica y el ojo del paciente pueden variar a lo largo de la prueba, lo que implica un potencial incremento de variabilidad de las mediciones realizadas, dependiendo la fiabilidad de la prueba en gran parte de la colaboración del paciente y de la experiencia del explorador $(2,3)$.

La tomografía óptica de coherencia (OCT) es una técnica no invasiva inicialmente planteada para el estudio de las enfermedades del polo posterior. Sin embargo, algunos autores han sugerido la posibilidad de utilizar dicha técnica para el estudio de las estructuras del ángulo esclero-corneal, mostrando ser una técnica confortable y de gran resolución espacial a este nivel anatómico (4).

En este artículo, mostramos el uso del OCT 3 para la visualización del segmento anterior,
El estudio se realiza sobre 5 pacientes con diversas patologías a nivel del segmento anterior: 2 pacientes con glaucoma agudo de ángulo cerrado, 1 paciente con rubeosis iridis, 1 paciente con un traumatismo penetrante ocular causante de una iridectomía y 1 paciente con un quiste estromal primario del iris.

En todos los casos, se obtienen imágenes del segmento anterior mediante BMU (OTI Systems ${ }^{\circledR}$, sonda de $35 \mathrm{Mhz}$ ) y Stratus OCT 3 con fines diagnósticos así como para el control de la evolución. La obtención de imágenes mediante OCT 3 es posible gracias a las distintas propiedades ópticas de las estructuras del segmento anterior. Para conseguir dichas imágenes con el OCT, se debe enfocar el limbo corneal mediante la ruleta de defecto refractivo (tendiendo hacia valores positivos), hasta obtener una imagen nítida de la zona a estudiar. Una vez enfocada la zona, se selecciona el protocolo de estudio «Line»y se procede al escaneado de la misma. En todo momento, se muestra el área que está siendo escaneada por el OCT en la cámara en tiempo real del aparato, pudiendo modificar en cualquier instante el área a estudiar. Se pueden realizar capturas en todas las posiciones horarias, siendo más sencillas las regiones horizontales nasales y temporales. 


\section{RESULTADOS}

\section{Caso 1}

Mujer de 58 años que acude de urgencia a nuestras consultas por dolor ocular unilateral de unas horas de evolución acompañado de cierto grado de disminución de agudeza visual. A la exploración presenta inyección ciliar, midriasis media arreactiva y una presión intraocular de $53 \mathrm{~mm} \mathrm{Hg}$. Ante este cuadro se establece el diagnóstico presuntivo de ataque agudo de glaucoma de ángulo estrecho y se procede a la toma de imágenes mediante BMU y OCT. El OCT muestra un cierre angular con contacto entre la malla trabecular y la zona más periférica del iris en los cuatro cuadrantes (fig. 1). De igual forma la BMU muestra una disposición similar del ángulo en los $360^{\circ}$ (fig. 2). Se instaura el tratamiento médico correspondiente para posteriormente realizar unas iridotomías bilaterales con láser YAG. Tras las iridotomías se realiza un OCT de cámara anterior que nos muestra como la iridotomía en el ojo del ataque agudo de glaucoma no es permeable, siendo necesario retratar la zona con nuevos impactos (fig. 3). Tras este segundo tratamiento se realiza un nuevo OCT que, esta vez sí, muestra permeabilidad de la iridotomía, apertura del ángulo y profundización de la cámara anterior (fig. 3).

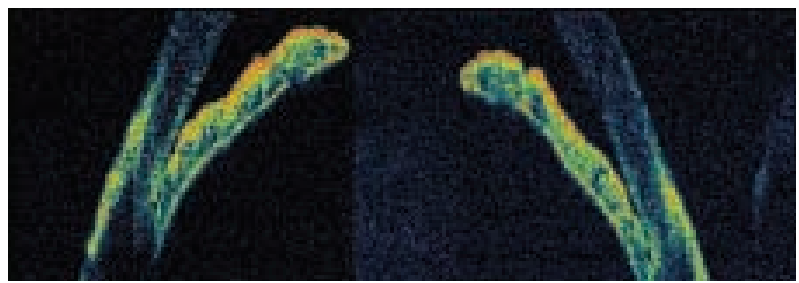

Fig. 1: Cierre angular mostrado mediante OCT (caso 1).

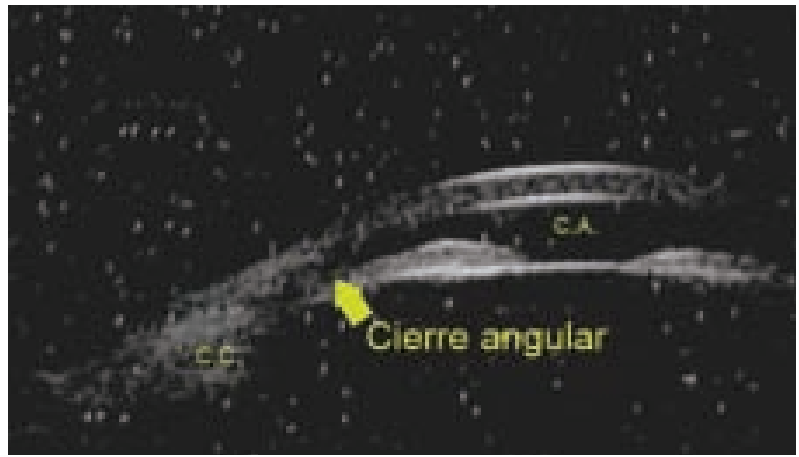

Fig. 2: Cierre angular mostrado mediante BMU (caso 1).

\section{Caso 2}

Mujer de 45 años remitida por Medicina Interna para estudio de fondo de ojo por presentar midriasis media bilateral con cefalea, pérdida de visión, náuseas y vómitos de 12 horas de evolución. A la exploración se objetiva una agudeza visual de 0,8 en ambos ojos y una midriasis bilateral arreactiva; el resto de la exploración, incluídas las papilas, es normal, salvo que presenta cámaras anteriores estrechas y unas presiones intraoculares de 65 y 58 $\mathrm{mm} \mathrm{Hg}$ en cada ojo. Ante este cuadro se procede a tomar imágenes del polo anterior con OCT (fig. 4) y BMU (fig. 5), mostrando un cierre angular en los $360^{\circ}$ y de forma bilateral. Con estos datos, se establece el diagnóstico de ataque agudo de glaucoma bilateral, se prescribe tratamiento médico para dicho cuadro y se realizan iridotomías bilaterales que resultan permeables como muestran las imágenes del OCT, ampliándose la profundidad camerular en ambos ojos (fig. 6).

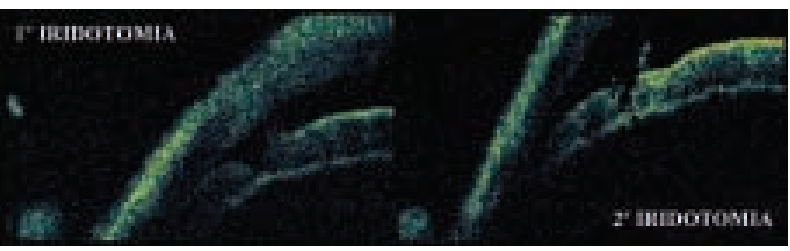

Fig. 3: Valoración de la permeabilidad de las iridotomías mediante OCT (caso 1).

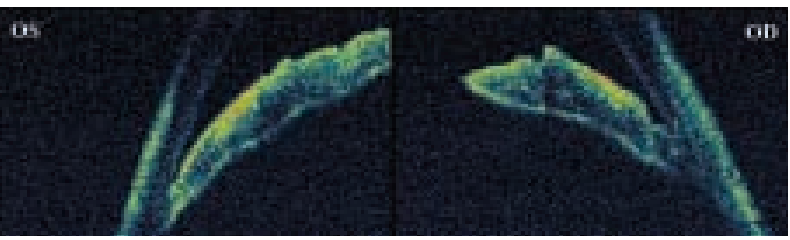

Fig. 4: Cierre del ángulo esclero-corneal visualizado mediante OCT (caso 2).

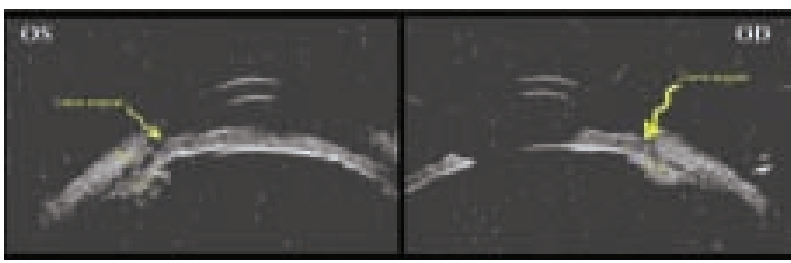

Fig. 5: Cierre angular bilateral mediante BMU (caso 2). 


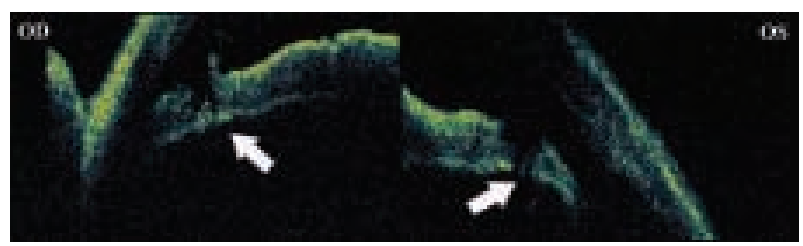

Fig. 6: Iridotomías permeables (flechas blancas) y ampliación de la cámara anterior (caso 2).

\section{Caso 3}

Varón de 45 años, diabético tipo I desde hace 14 años, diagnosticado de retinopatía diabética no proliferativa severa en la última revisión oftalmológica. Acude a su revisión habitual mostrando una intensa rubeosis iridis en el ojo izquierdo. Se toman fotografías del segmento anterior y se realiza un OCT de iris que muestra una señal hiperreflectante en la superficie externa del iris, correspondiente al área de neovasos (fig. 7). En este caso, el OCT muestra que la configuración del ángulo esclero-corneal es abierta. Posteriormente, el paciente es tratado mediante panfotocoagulación retiniana.

\section{Caso 4}

Mujer de 38 años que acude de urgencias por presentar un traumatismo penetrante ocular por un fragmento de una pinza de ropa. La paciente muestra una perforación a nivel corneal con afectación del iris y del cristalino. Se interviene de urgencias realizándose la extracción del fragmento y posteriormente, el cierre corneal y la facoemulsificación del cristalino con colocación de una lente intrasacu-

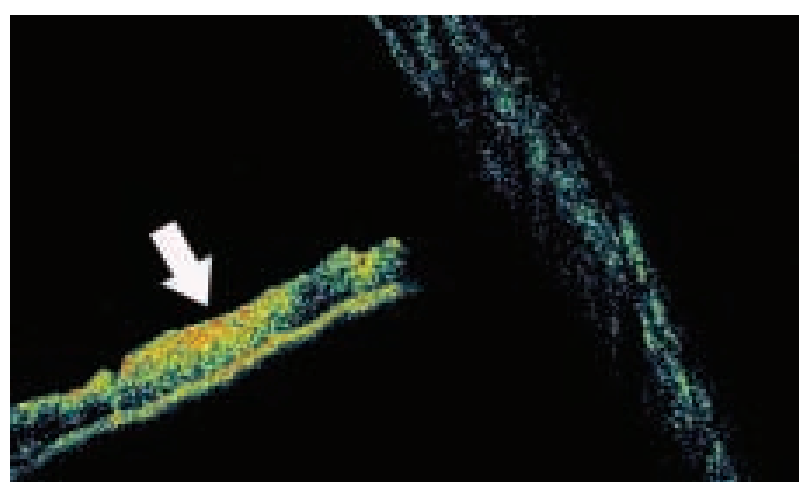

Fig. 7: Hiperreflectividad iridiana en área de neovasos (flecha blanca). lar. En una revisión posterior a los 5 meses de la lesión, se toman imágenes del segmento anterior mediante OCT (fig. 8). El OCT muestra claramente la zona de la iridectomía traumática, así como la disposición de la lente intraocular.

\section{Caso 5}

Varón de 34 años que acude a la consulta remitido por optometrista para valorar tumoración a nivel iridiano sin repercusión funcional. A la exploración se objetiva una formación sobreelevada de aspecto quístico y contenido lechoso a nivel del iris, cerca del reborde pupilar. No refiere ningún antecedente oftalmológico de interés. Se obtienen imágenes con BMU y OCT y se establece el diagnóstico de quiste estromal primario de iris (figs. 9 y 10).

En todos los casos anteriormente mencionados, el tiempo medio de exploración utilizando ambos instrumentes varío considerablemente, de esta forma, mediante el OCT se lograron exploraciones completas del segmento anterior en tiempos inferiores a 5 minutos. En cambio, la BMU, fundamentalmente debido a la preparación previa que conlleva, así como a la mayor dificultad para localizar la lesiones a estudiar implicó tiempos de exploración superiores a 10 minutos.

\section{DISCUSIÓN}

El glaucoma agudo de ángulo estrecho constituye la principal forma de glaucoma en la población

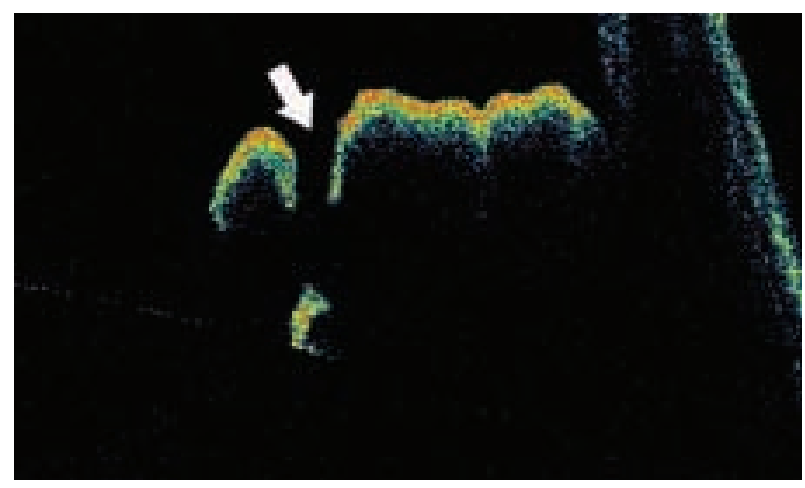

Fig. 8: OCT mostrando una iridectomía traumática (flecha blanca), así como la opacificación de la cápsula posterior. 


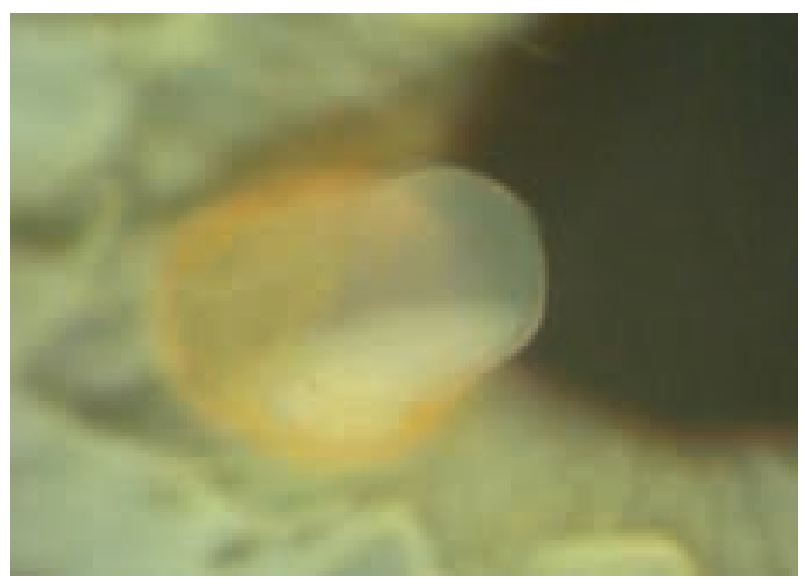

Fig. 9: Quiste estromal primario de iris.

asiática; sin embargo, es una entidad más rara en pacientes caucásicos. Actualmente, la gonioscopía continúa siendo la técnica de elección para la visualización del ángulo, siendo indispensable en la exploración de los pacientes glaucomatosos.

En la última década, el desarrollo de la BMU ha permitido mejorar la visualización de las estructuras de la unión esclero-corneal, aportando imágenes con una alta resolución espacial. Sin embargo, la BMU continúa siendo un método exploratorio poco extendido, debido en parte a ser una técnica poco confortable para el médico y el paciente, y con una variabilidad no despreciable en manos no expertas.

La tomografía óptica de coherencia, a diferencia con la BMU, es un método diagnóstico no invasivo ampliamente extendido, debido a sus múltiples aplicaciones para el estudio de las enfermedades retinianas.

Sin embargo, el empleo del OCT para la obtención de imágenes del segmento anterior ha sido poco estudiado en la literatura, existiendo pocas referencias en la misma sobre su utilidad.

En los casos de glaucoma agudo de ángulo estrecho (casos 1 y 2), el OCT permite una rápida valo-

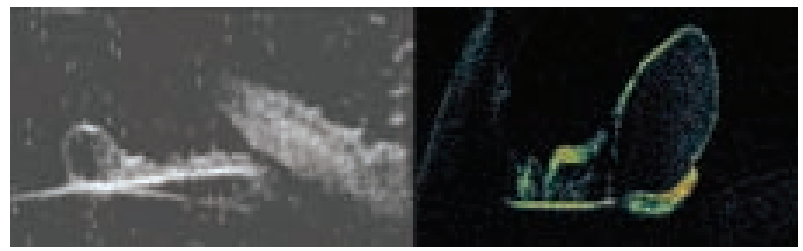

Fig. 10: BMU y OCT de un quiste estromal primario de iris, observándose el contenido lechoso en ambas exploraciones. ración del grado de oclusión angular y de la morfología del iris, pudiendo obtener imágenes rápidamente en cualquier meridiano. Previamente, otros autores (5) han mostrado que cuanto más cerrado es un ángulo mejor es su visualización con OCT, permitiendo observar la raíz del iris en casos de ángulos ocluídos. En nuestros casos se muestra claramente este hecho de forma que, cuando el ángulo está cerrado puede visualizarse la raíz del iris y su contacto con la malla trabecular pero, tras la realización de las correspondientes iridotomías, se amplía la cámara anterior y surge un área no reflectiva a ese nivel, indicando la apertura angular. Además, en estos pacientes con cierre angular, el OCT permite una rápida evaluación de estado de las iridotomías realizadas, ya que la cámara en tiempo real permite localizarlas y escanearlas con facilidad. Con el OCT podemos obtener información sobre el grado de profundidad y permeabilidad de las iridotomías, así como del grado de apertura angular logrado tras las mismas. Así, puede observarse que en el caso 1, el OCT permite conocer que la primera iridotomía fue incompleta, con la cámara anterior muy estrecha, visualizándose la raíz del iris. En este caso, fue necesario un retratamiento que permitió que la cámara anterior se ampliase, adquiriendo una configuración abierta identificable con el OCT. Además de la valoración del ángulo esclero-corneal, el OCT puede ser utilizado para el estudio y evaluación de diversas patologías iridianas. De este modo, los casos 3, 4 y 5 muestran distintos ejemplos en los que se ha empleado el OCT para analizar el iris.

El caso 3 representa un ejemplo claro de rubeosis iridis por retinopatía diabética proliferativa. En este caso, el OCT muestra una hiperrefringencia en la cara anterior del iris, correspondiente a los neovasos, junto con una disposición abierta del ángulo.

En el caso 4, el estudio mediante el OCT permite visualizar con facilidad la iridectomía traumática ocasionada por la entrada intraocular de un fragmento de pinza de ropa. En este paciente puede observarse una línea hiperrefringente en la zona del reborde posterior de la lente intraocular debido a la opacificación de la cápsula posterior del cristalino.

El caso 5 corresponde a un paciente diagnosticado de quiste estromal primario de iris. El quiste estromal primario de iris normalmente presenta un crecimiento lentamente progresivo, hacia la cámara anterior, pudiendo ocluir incluso el eje pupilar (6). Tradicionalmente se ha denominado quiste estromal de iris por su localización anatómica, aunque 
realmente está constituido por una pared de epitelio de tipo conjuntival, por lo que el término estromal, desde el punto de vista histológico, parece poco apropiado (7). Este quiste suele ser diagnosticado en la infancia, pudiendo localizarse en cualquier cuadrante. El quiste suele contener un fluído de aspecto claro, semitransparente, permitiendo la visualización del epitelio iridiano posterior a la lesión.

En este caso, estudiamos la lesión mediante BMU, permitiendo comprobar el aspecto quístico, su contenido y cuantificar su tamaño. El estudio con OCT también permitió analizar la anatomía del quiste, alcanzando un nivel de detalle estructural superior al obtenido mediante biomicroscopía ultrasónica, sin embargo, al carecer de un software adecuado para polo anterior, no es posible medir el tamaño real de la lesión, lo que no impide detectar un posible crecimiento en sucesivas revisiones siempre y cuando la línea del scan se vuelva a situar en la misma localización.

El OCT permite la obtención de imágenes de alta resolución $(<10 \mu \mathrm{m})$, superior incluso a las obtenidas mediante la biomicroscopía ultrasónica (25 $\mu \mathrm{m})$. Por otra parte, el tiempo en la generación de las imágenes es mucho más rápido con el OCT (400 escáneres por segundo) que con la BMU convencional (8 imágenes por segundo) (4).

No obstante, la principal ventaja del OCT frente la BMU convencional radica en que la tomografía es un método de no contacto, y que no requiere una posición supina del paciente. Esto permite que la prueba sea mejor tolerada por los pacientes, siendo más confortable que la BMU convencional.

Una desventaja del OCT frente a la BMU es la incapacidad en la obtención de imágenes del cuerpo ciliar, zónula y cristalino, ya que el epitelio pigmentario del iris ejerce un efecto pantalla para el haz de láser impidiendo la visualización de dichas estructuras. Actualmente, el láser de $820 \mathrm{~nm}$ del OCT 3 es incapaz de mostrar el cuerpo ciliar, aunque si bien se están desarrollando tomógrafos con distintas longitudes de onda que pueden llegar a mostrarlo (8). La BMU también ofrece ventajas sobre el OCT cuando pretendemos realizar un estudio del ángulo esclero-corneal en pacientes con opacidades de medios a nivel corneal y limbar. En estos casos, el láser del OCT no logra penetrar en la cámara anterior y es incapaz de escanear estructuras del ángulo esclero-corneal y del iris.

En conclusión, el OCT 3 a pesar de no estar diseñado específicamente para este uso, puede servir para obtener imágenes del ángulo esclero-corneal así como de otras estructuras del segmento anterior, constituyendo una exploración no invasiva, relativamente cómoda y segura para el paciente.

\section{BIBLIOGRAFÍA}

1. Pavlin CJ, Harasiewicz K, Sherar MD, Foster FS. Clinical use of ultrasound biomicroscopy. Ophthalmology 1991; 98: 287-295.

2. Tello C, Liebmann J, Potash SD, Cohen H, Ritch R. Measurement of ultrasound biomicroscopy images: intraobserver and interobserver reliability. Invest Ophthalmol Vis Sci 1994; 35: 3549-3552.

3. Urbak SF, Pedersen JK, Thorsen TT. Ultrasound biomicroscopy. II. Intraobserver and interobserver reproducibility of measurements. Acta Ophthalmol Scand 1998; 76: 546-549.

4. Leung CK, Chan WM, Ko CY, Chui SI, Woo J, Tsang MK, et al. Visualization of anterior chamber angle dynamics using optical coherence tomography. Ophthalmology 2005; 112: 980-984.

5. Moreno J. Aplicaciones de la tomografía óptica de coherencia en el estudio del ángulo camerular. In: Teus MA. Atlas de gonioscopía en el glaucoma por cierre angular. Madrid: Allergan SA; 1999; 151-162.

6. Lois N, Shields CL, Shields JA, Mercado G, De Potter P. Primary iris stromal cysts. A report of 17 cases. Ophthalmology 1998; 105: 1317-1322.

7. Shiels JA, Shields CL, Lois N, Mercado G. Iris cysts in children: classification, incidence, and management. The 1998 Torrence A Hakley Jr Lecture. Br J Ophthalmol 1999; 83: 334-338.

8. Radhakrishnan S, Rollins AM, Roth JE, Yazdanfar $S$, Westphal V, Bardenstein DS, et al. Real-time optical coherence tomography of the anterior segment at $1310 \mathrm{~nm}$. Arch Ophthalmol 2001; 119: 1179-1185. 